\title{
The Structure of Musical Communication
}

\author{
Alexander Yakoupov \\ Russian State Specialized Arts Academy \\ Moscow, Russia \\ e-mail: rgsai@mail.ru
}

\begin{abstract}
The article is dedicated to the problem of the theory of mass communication in music. The author specifies three major communication types and analyses communicative spheres of a composer, a musician and a performer. A triad of the main spheres is described and characterized here.
\end{abstract}

Keywords-music communication; mass listener; accumulation; generation; presentation of music; metastructural communication level

\section{INTRODUCTION}

The structure of the integral process of musical communication is very complicated and diverse, as well as the process itself, the process dynamically evolves within the social time and space. The structure has to fully encompass the open and closed channels of information that function on different levels and connect all the participants of the musical process (individual, collective, depersonalised social entities like 'public consciousness', etc.) both to the sources of information, its senders (as well as keepers) and to the those who assist in transforming the musical information into other kinds of human creativity and activity in general.

Let us first define the major levels of the informational communication. We believe that one might single out three such levels. They are distinguished by their links and connections, their position in the integral structure, the specific character of the information that circles through them. The levels are: the structural (central), the microstructural and the meta-structural.

The central - the structural level - includes the major participants of the musical process and the main channels of information that sustain the functioning of the system as a whole. One might distinguish four major spheres of communication here. The first one is the sphere of creating the musical values by the author (the individual and the collective composer); the second one is the sphere of the artistic recreating the author's work by the performer (the individual and the collective performer); the third sphere is the sphere of the active perception (by the listener); the fourth is the sphere of the aesthetic and socio-cultural evaluation of both the results of the author's and the performer's activity, and all the social phenomena, actions and events that have to do with the musical art (the musicologist-critic, and, to a degree, the individuals that deal with the functioning of the musical process and managing it - the producers, the administrators, the managers, sponsors, etc.).

The structural level provides for the functioning and developing of the integral communicative process within the musical art. The information that circulates through the communicative channels on this level is contained, basically, in the open musical codes - the codes that, in accordance with the traditions, the norms and the musicians' skills, become open to deciphering by every participant of the process, the deciphering that implies the constant re-creation of the isomorphic form of the original information (unchanged, enriched or reduced).

Each of the cited communicative spheres contains a particular micro-structural level that provides for the encoding and decoding of the aesthetic information, for the connection with the other spheres of communication as well as for the transfer of the skills and traditions that are developed. One also has to note that, together with the general informational structures, each of the spheres possesses its certain specific means of storing, transmitting, encoding and decoding information in accordance with the diverse material that defines the local character of a given sphere.

Finally, the third - the meta-structural - level provides for the inclusion of the musical process into the wide area of the diverse connections to the culture of the society as a whole, the social context of art, the creative and active nature of man. It is here that the communicative chain is locked, the communicative circuit is closed providing for the functioning and the effectiveness of the musical process in a society.

The information that circulates on the meta-structural level possesses the most diverse character. The mechanism of its encoding and decoding takes on a largely secondary importance. The transfer of information in large structural units comes to the foreground; the units are the patterns, typological constructions, traditions, the emerging norms, etc. - the units that often circulate through the hidden channels, through the structures of the public consciousness, the structures through which the cultural values are absorbed.

It is here that the communicative processes may be most open to the influence from the society and its institutions, impacting, in their turn the most basic — the structural level of the whole process of musical communication. While 
venturing a more thorough analysis of the ways the information circulates at this level, let us first define the basic relationships between the four cited spheres. The initial, the fundamental sphere is here the sphere of the composer. It gives the initial impulse to the whole process and bears the major informational charge, since this is where the musical form is created, the form in which the author's aesthetic idea is specifically encoded for further transfer through the channels of information.

The composer's sphere is connected by a direct information channel to the performer's sphere, since the latter is an indispensable participant of the musical process, the author's 'co-creator', so to speak; he deciphers the original author's form and creates in his interpretation his own performer's form, the code directed towards the listener in accordance with the latter's perceptive capabilities and active artistic absorption of the received information.

Decoding the information received from the composer and the performer, the listener, in his turn, creates in his artistic imagination his individual variant (invariant) of the original aesthetic meanings. An isomorphic re-creation of both the composer's and the performer's original intention takes place here, in the circumstances of a certain differentiation of the meanings. This becomes possible due to the comparisons made between different performances, due to a deeper familiarity with the style of the composer, or this or that performer. In the listener's sphere the evaluation of the received information first takes place. That evaluation is transmitted through the information channels to all the other participants of the process and influences its further development.

Finally, the last sphere, the sphere of the musicologistcritic - receives the information from the previous three spheres. Then it is subject to study and expert evaluation at a professional level, when the opinions of all the participants are taken into consideration. The evaluation includes personal opinions and preferences that are characteristic of individual perception, and this sometimes leads to a certain deformation of the actual aesthetic meaning of the information received (this is the 'static' that brings about the 'distortions' of the original message).

The evaluation criteria that are developed in this sphere may perceptibly influence all the other spheres of the musical process, their aesthetic aspect, stimulating or inhibiting some of the aspects of the process - regulating it to a degree. The impact of the various structures that are active in organising the musical life - the press, the radio, the television, etc. - may also be said to belong to this sphere.

Therefore, one may state that the communicative spheres of the composer, the performer, the listener and the musicologist-critic interrelate and co-operate. The central core of their intersection is the musical form that is initially created by the composer, that bears the encoded ideal information and is therefore the initial point for decoding, artistic re-decoding and evaluation by all the other participants of the process of communication.
The structures of the communicative processes at all the other levels of the communicative system are largely analogous. The micro-structural level of the informational interaction will be considered further within the analysis of each of the spheres of the central structural level. The metastructural level - the entrance of the musical process into the wide socio-cultural context - will be considered in the closing paragraphs of the present chapter.

It is only recently that the complexity of the process of musical communication became understood, as well as the existence of the certain methods of encoding and decoding information, the open and hidden channels of transmitting and receiving information, the importance of the isomorphic principle in restoring the original meanings, etc. The works on the general theory of communication were largely instrumental in bringing about that understanding. So, it seems necessary to touch upon some of the works, at least briefly.

Two different notions of the process of information interchange have emerged in the national and foreign theory of communication by the end of the current century. One of them was formed within the humanitarian plane (V. Borev and A. Kovalenko, Y. Lotman, Lasswell, Lewis, LeviStrauss, R. Jacobson and others), the other - within the scientific (N. Wiener, W. Meyer-Eppler, A. Moles, Shannon, Ashby and others). Each of the trends offers its own methodology and terminology, theoretical approaches and solutions.

But the representatives of the both trends are united in their approach to structuring the process in question. They all utilise the method suggested by Lasswell in the 30s and $40 \mathrm{~s}$. He suggested that the structure of the process of communication has to be cleared up by the use of the questions like who informs? who transmits? who is informed? to whom is the communication addressed? what is the communication (message)? etc. Then each one of the meaningful parts of the structure found in this way should be theoretically analysed.

Utilising this method for a thorough analysis of the process of musical communication, one finds that, on the one hand, the process is characterised by an integrity and completeness of the cycle and a constant expansion of the process boundaries, and on the other hand, the process is characterised by the quadratic property of the basic, central structure where the gradual production, transfer and restoration of the original aesthetic meanings takes place.

At the same time the development of the process of musical communication at the meta-structural level, its contact with the wide socio-cultural practice, the hidden character of many channels of information that influence the process indirectly with a considerable lag in time - all that and many other peculiarities of the communicative structure in question, let alone the complexity of the aesthetic information and the spontaneous development of the musical art itself, lead us to a cautious approach to the various analytic procedures gleaned from other branches of theoretical and scientific thought, to the belief that the 
results gained by using such procedures should be evaluated taking into consideration the specific character of art.

Therefore, before analysing the integral structure of the process of musical communication we should give our attention to the following important considerations. The closed character of the cycle that the system possesses emerges as the result of the many feedback links and connections that work upon different structural levels and, to a great degree, account for the complexity of the system as a whole.

The integrity of the system prevents the communicative area of music from being dissected into separate and sufficiently closed constructs. It proves to be impossible to stop the flow of the process or 'take out' any of its parts without destroying the whole of it. Thus the structural method according to Lasswell, as well as the questionnaire he suggested, hardly work here, since in analysing musical communication one cannot separately consider the problems of transmitting, encoding and receiving information because the final results of a study within this method do not correspond to the object's degree of complexity.

Therefore we believe that it would be more worthwhile to try and study the structure of musical communication in relation to the positions that are taken by the participants of the process themselves, taking into consideration at least the basic informational channels as well as the specific character of encoding and decoding of the original meanings transferred through the channels. Thus, in case one of the participants is in the position of the sender of ideas, and therefore in position to encode them, the three remaining participants (or at least one of them, if we take into consideration the quadratic character of the structure) would take the position of receiving the information, decoding the original meanings. Within this logic, it would be easier to define the active and passive elements of the process that interact at a given moment of time; it would be easier to follow the dynamics of the basic links between the information channels. Thus the understanding of the general structure of the system of musical communication would be more approachable.

We would also like to make the following preliminary statement. In the musical process, not every communication is personified. In the remote past, in the era of syncretic art they were all practically levelled. So, the impersonation of certain communicative links still has its place in present day music.

We should also single out another important channel of communication that provides for the evolutionary development of music. It is the system of communication within the musical creative activity itself. Both the channel of personal, individualised transfer and the channel of impersonal transfer might be distinguished here.

The former consists in the system of teaching to create, perform, record and publicise the musical art in a society, the system of preserving the art's traditions (the musical education, the mass propaganda of music in kindergarten and school, in different clubs; lectures and discussions on the musical art, the meetings with the musicians).

The impersonal transfer may be said to consist in the invisible process of the spontaneous development of the musical language, the form of the different expressive means (the emergence of the intonational and rhythmic formulas, cadences, different forms of the content embodiment, the typological genres, the emergence of the 'intonational vocabulary' of an epoch, etc.). The development and the different types of encoding and decoding the text (the ciphered bass, melismatic, the contracted notation, diminution, improvisation, etc.) may be said to belong here, as well as the ways and means of impacting the listener, etc.

For instance, the very process of folklore development is of an impersonal (or rather, personal/impersonal) nature. The figure of the composer as the individual translator of the musical ideas does not appear here, the collective creator and listener are often merged together, and the process of communication itself largely takes place in a hidden manner.

\section{THE COMPOSER'S SPHERE}

The structural analysis of the musical communication should really be started from the composer's sphere, since it is the composer who gives the whole process the initial spur, the initial impulse by creating the musical values.

The appropriate chart shows just one facet of the process "Fig. 1", related directly to the musical form. At the same time, in the theory of communication the figure of the creator of music should be regarded in a much broader fashion. The sphere should reflect the diverse factors that influence the creative activity, the various sources of the creative ideas' accumulation, and the diversity of the composer's relations with the social institutions of the functioning and publicising of music. Only such a thorough reflection of the composer's participation in the cultural sphere provides for the discovery of the major links and interrelationships, for finding the general regularities and tendencies of the process development.

changes in the essence of the communicative processes. In relation to this, one might cite a fact from $\mathrm{S}$. S. Prokofiev's biography. In January 1922 he was finishing a tour of the US, depressed by the fact that the American public lost interest to his work. (During his last concerts, upon his impresario's insistence, he mostly performed work by Moussorgsky and very few of his own pieces). But when he returned to tour the United States in 1925 he was astonished by the changes that took place. That time he was accepted as an established celebrity and was asked to perform mostly his own pieces. It is noteworthy that he, with the Koussevitzky orchestra, had to perform his 3rd concerto three times. V. Delson noted that after the tour Prokofiev himself admitted that he 'had completely lost any hope of ever coming to understand the reasons for success or failure' $[2,109]$. 

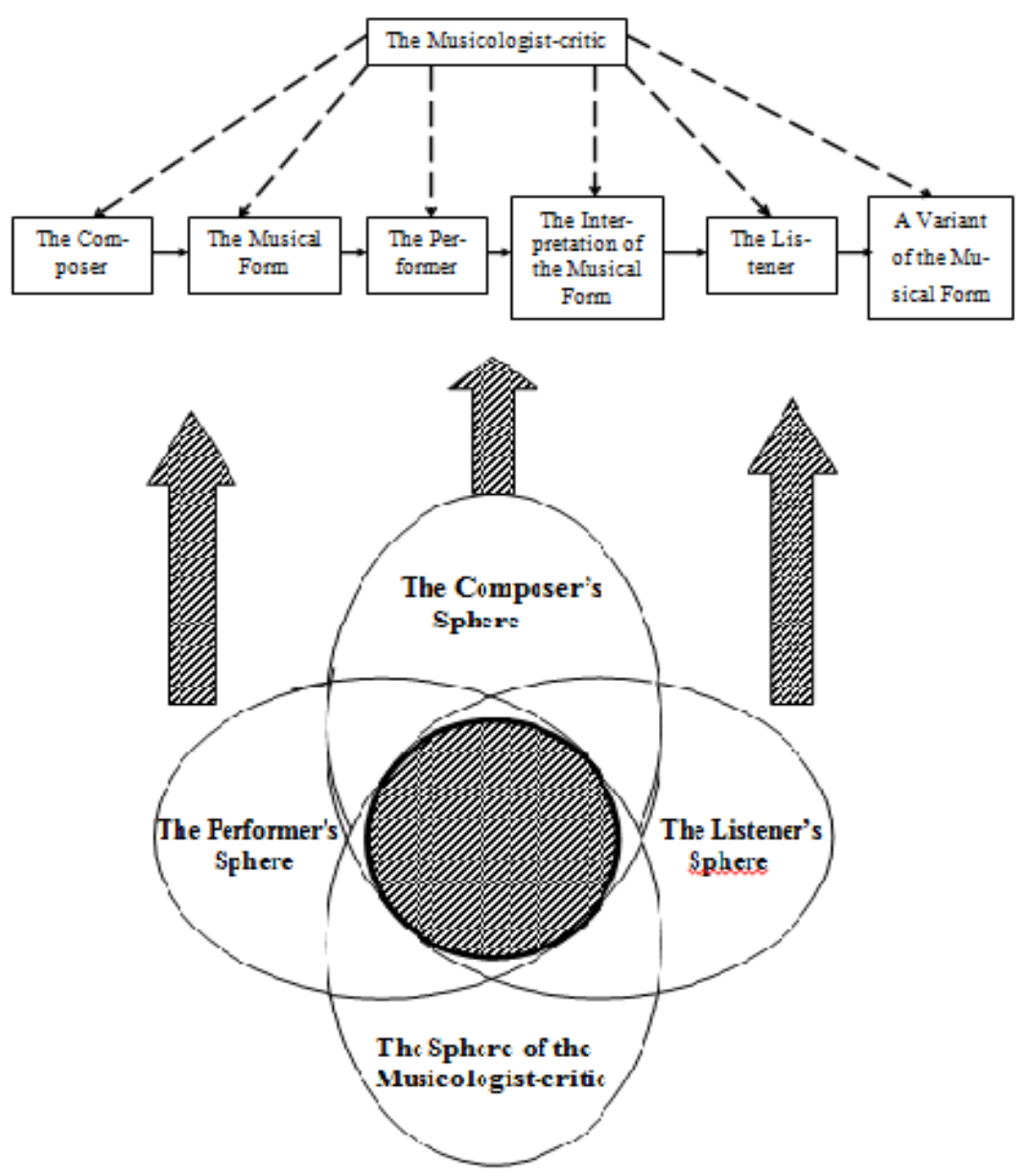

Fig. 1. The Musical Communication Process

Each of the four cited spheres of the scheme of the communicative system of music "Fig. 2", including the composer's sphere is characterised by its own part in the public musical consciousness. In practice it is manifested in the discrepancies in views and tastes, in the differences in approach to the musical phenomena, in the criteria of their evaluation.

Even the most talented and independent composer would not be able to create by sheer virtue and power of his talent, without drawing on his past experience and the reality around him. He is deeply related to the social practices and the musical consciousness, his environment and the human culture as a whole. Had he even been able to create something completely new, the very fact of his controversy with the traditions of the past and the norms of the present would have been seen as an aesthetic dispute with those traditions and norms.

It is characteristic that all the factors that influence an artist are at the same time the sources for the artistic ideas' accumulation. Say, R. Shchedrin when asked of the essence of the term 'the accumulation of artistic ideas', replied 'it is not only the musical ideas which I mean here; not only definite themes or sound images; I mean everything that we perceive, see, hear, inhale, sense or touch ... All that is subconsciously processed by a musician ... and is eventually reflected in his musical creation. Sometimes it happens that a Renaissance or a present-day painting is suddenly related to a certain musical phrase, intonation, sequence of sounds'. $[1,226]$.

Therefore, all that is included into the communicative field that connects the composer to the performer and the listener and the complex phenomenon of the public consciousness. It is formed throughout the whole history of the musical culture by all the participants of the communicative process, first of all, by the composer. It is possible to think, after a cursory glance, that the formation of such consciousness proceeds spontaneously, sometimes chaotically. An infinite number, not only of different musical creations, but of general and particular ideas and notions, thoughts and feelings that arise in musical practice become a part of the public consciousness and influence the inner world of the artist and impact all the other participants of the communicative process.

In practice, we often encounter the great unpredictability of the very process of the formation of the public musical consciousness. And that, of course, leads to significant 


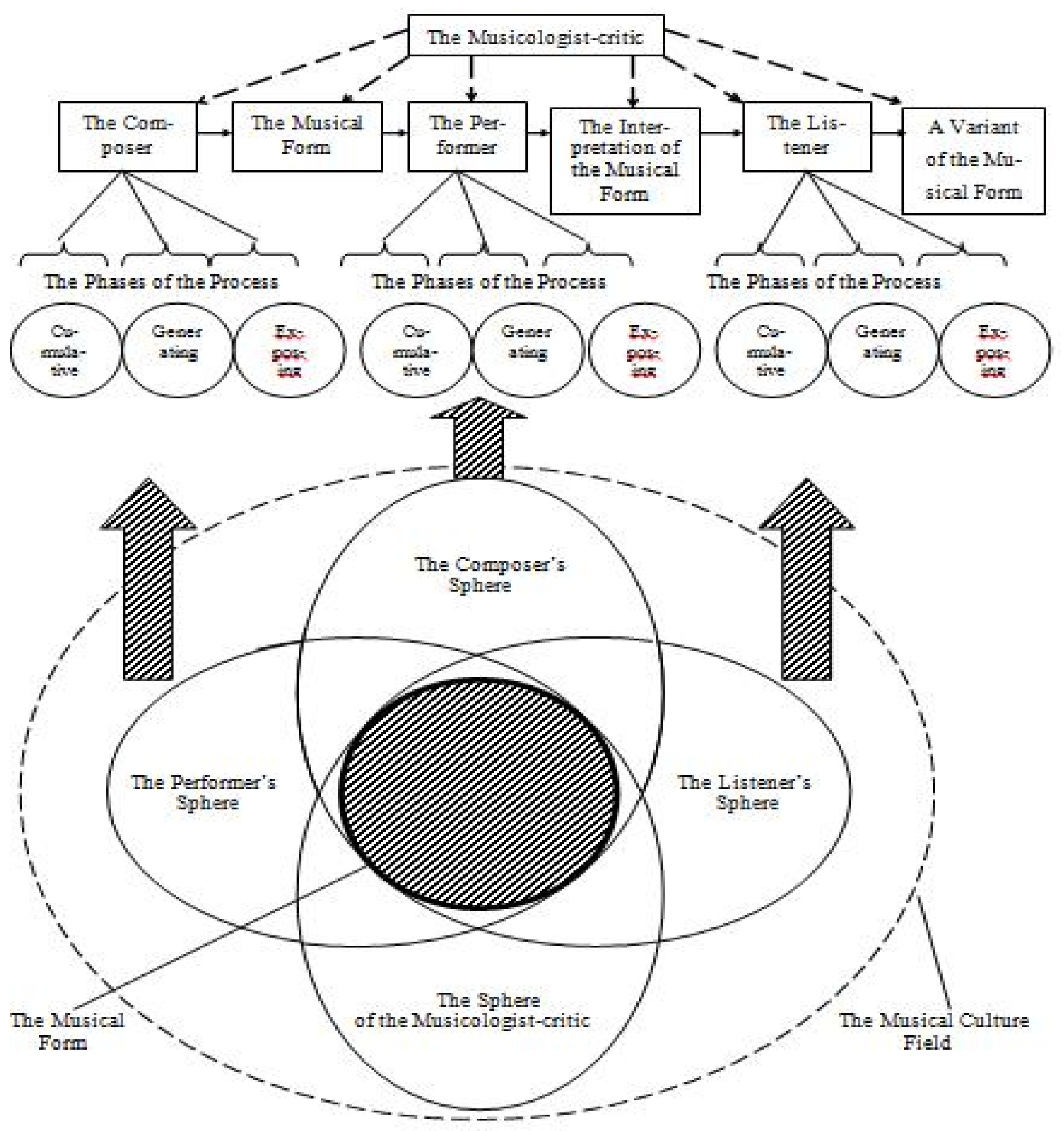

Fig. 2. The Basic Scheme of the Structure of the Musical Communication Process 
Often times, the established leaders of the composing community fail to be quite as popular among the general public. And vice versa, there are numerous cases when a widely popular musician fails to get quite as high validation from his professional colleagues. At the same time the history of music has seen a number of instances when communication took place between the remote areas of the public musical consciousness. Thus, after the Russian revolution, the psychological subtlety of the musical phrase and the refined culture of feelings, so characteristic of Scriabin's work, had called forth response and understanding both from the composing community and the general public.

This example, being very well known, might be considered banal, but for one circumstance. It seems that there is some possibility of foreseeing such instances by following the tendencies of the public musical consciousness development and the regularities of the development of the communicative processes, in particular, their dependence upon the psychological aspect of musical perception, the conditions of the purposeful formation of the listeners' interests.

From the viewpoint of the musical communication theory the cited situations with the music by Scriabin and Prokofiev may serve as a good example of the conflict between the content of their pieces and the possibilities of communication that were accounted for by the public needs in that respect. The specific character of these masters' creations implied an intellectual contact with the listener. Thus the conditions of the 'reproduction' (Hegel) of this music demanded, say, in Scriabin's case, small chamber concert halls, and in case of both Scriabin and Prokofiev it had to be directed precisely to a certain addressee. But the listeners caught the wind of this new style of perception by sheer chance. This style turned out to be essentially akin to the feelings and attitudes of the masses of that epoch.

In short, the composer's sphere is sufficiently complex and diverse. At least three phases may be singled out in its structure. They are closely connected but characterised by different functions and directing vectors. The phases are the cumulative, generating, and exposing. They interact both in a sequential and parallel manner. At each given stage they define the highest step in the development of the communication and its direction.

The cumulative phase of the process. At the time of accumulating the aesthetic potential and artistic ideas the author rather 'consumes', draws upon the sources - the life and culture of a society - than contributes to them. The development of the communicative process in this case is largely unidirectional, that is, evolves from the society towards the artist.

It would be more precise to say that the period of accumulating the aesthetic ideas lasts throughout the whole life of an artist. Only its intensity changes. When the work is being created the accumulation weakens greatly. When the work is completed, there's a moment of an artistic crisis, 'exhaustion'. As a result, the composer needs to be 'recharged' with new ideas. So, it is largely impossible to define, even with relative precision, the temporal, the deep boundaries within which the intensity of the cumulative process fluctuates, since the individual characteristics of different composers vary a great deal. It would also be a mistake to regard the author of a musical piece as a person that, in contrast to the other participants of communication, mostly 'gives' or donates to the society. The composer's talent is also a talent of accumulating ideas, the talent of artistically processing the various information flows that are produced by the society itself.

The source and the basis of the musical communication at the cumulative phase is contained in the culture in general, the culture that is created by the public consciousness on the one hand and by a much broader scope of determinations on the other. And upon this general field, each kind of art bears its own function, carries out both specific, characteristic tasks, and wider (within culture in general) functions that permeate all the layers of the consciousness and the culture.

Regarding this complex process, one might say that definite kinds of art might dominate a given epoch. Say, the ancient Greek art was dominated by architecture and sculpture that embodied the most lofty of the contemporary ideals of man and world. It is not by chance that the major criterion of the aesthetic validation was at that time contained in the 'human measure'. The Enlightenment puts literature in the foreground. The drama was then ruled by the principle of the triadic unity of place, time and action - a sign that the social space comes into the limelight in art. The moral principles of the society become the leading principles. In music, the dominance is then assumed by the vocal kinds and by the instrumental music that imitates the human voice and its intonational palette (the bow instruments culture, the violin music in particular).

There is a quite simplified hypothesis that the intensity and quality of the composer's artistic ideas is in direct proportion to the activity with which he draws them upon the social sources and to the quality of the ideas thus gleaned. We believe that the secrets of the ways the aesthetic impulses are determined are contained rather in the individual capabilities of the artist himself, in his capacity to perceive and process the extreme levels of the intensity and complexity of the information flow. The study of this complicated and, we believe, interesting problem is a matter of the future.

The idea of a piece first emerges in a certain inner (individualised) aesthetic field, as an idea, as some kind of infinity. Then it grows and evolves in the cultural field within the capacities of the aesthetic language that the epoch provides for the composer. But the composer often goes beyond this framework. When the next phase comes and the composer commits his work to a certain form, a code, the inner field that the creation sprung from still exists, in a contracted variant; it interacts with the form/code and facilitates its deciphering.

On the fact that a piece springs up spontaneously and integrally, within a certain artistic plane, S.V. Rachmaninov wrote the following, ' $<\ldots>$ there is every reason to believe 
that a composer possesses a great gift, for before creating he has to imagine. And his imagination has to be so strong that in his mind a whole picture of the future piece is created even before a single note is committed to paper. His finished work is an attempt to express the very essence of that picture in his mind through music' $[(3,560]$.

The field of the original creative idea is then embodied in the composer's remarks, partly expressed in his other creations; it includes his most diverse associations. It often serves as a 'general link', the major motif in his entire creative career.

The generating (creative) phase of the process. The second phase of the communicative processes in the composer's sphere is mostly related to the tasks of completing a piece and all the circumstances that facilitate its appearance.

The very readiness of the composer to produce a work is of primary importance here. The readiness may in its turn be considered as a specific dynamic condition of the inner information channel that links, artistically and communicatively, the cumulative and the generative phases of the composing activity. The inner aesthetic ideas that emerge constitute the strongest spur to creativity. But they spring up spontaneously, unexpectedly for the composer himself. It is this way that the ideas for the Sixth symphony, the Violin concerto, and some other works by Tchaikovsky emerged.

The fact that, while the piece is being created, the presence or absence of a definite order or demand for it is insignificant - this fact is proven by many masters. Thus, P.I. Tchaikovsky wrote to Mme von Meck, 'I would make an attempt to give You the general idea of the way I work. First of all, I have to draw a certain line between the two kinds of the works that I undertake:

the pieces that I write on my own initiative, because of a direct urge and an irresistible inner necessity;

the pieces that I compose led by some external impulse, upon a friend's or a publisher's request, or when I receive an order for composition, as the case was when I was asked to compose a cantata for the opening of the Polytechnic exhibition.

And I know from experience that the quality of the piece does not depend upon the division it belongs to...' [quoted by: 4 , v. 7, 117].

Alfred Schnitke was even more clear on that, 'I would say that the fact whether I receive an order for music or not is not of primary importance. It is important that it is born in accordance with its own specific laws. If it is so, I am ready to work on the ordered piece. If not, I'll decline the order' [5, 168].

Therefore the inner urge to realise a certain design, the urge that emerges as a result of a certain accumulation of information is always the decisive factor for the composer. At the same time one cannot disregard the importance of the external communicative links that connect the composer to the public musical consciousness and to the organisational spheres of culture. Thus, it is well known that V.V. Stasov, as the advocate of the patriotic ideas, produced a considerable impact upon the members of the Mighty Bunch (Moussorgsky, Borodin, Rimsky-Korsakov, et al) urging them to produce works related to the national themes. The Liszt circle in Weimar carried out a partly similar function. In the more recent times, one may cite the analogous impact of I.I. Sollertinsky's personality upon many Leningrad composers, D.D. Shostakovich in particular.

The performers that are close to the composer might also stimulate the creation of new work, since their urge for personal aesthetic realisation is often manifested in their need to be the first performers of the pieces dedicated to them. To their stimulation the musical art owes such masterpieces as the Mephisto-waltz dedicated to Tausig, Balakirev's Islamei dedicated to Nikolai Rubinstein, its first performer, the violin concerto by Brahms dedicated to J. Joachim, the vocal concerto by Glière, composed for D. Pantoffel-Nechetskaya, the cello concerto by Shostakovich, written for M.L. Rastropovich. etc.

The composer always tries to get in touch with those musicians who, he believes, might realise his original ideas, even enrich them to a certain degree. A. Schnitke remarked on that, 'I can't very well account for it, but I always have to establish some inner contact with the future performer. I should know who I compose for' $[6,168]$. And it is not by accident that Schnitke's work is meant for a definite circle of the performers that are more or less close to him. These are the violinists O. Kagan, G. Cramer, M. Lubotsky, the 'cellists M. Rastropovich, N. Gutman, the violist Y. Bashmet, the conductors K. Masur and G. Rozhdestvensky. The composer admitted that not only were they wonderful musicians, but distinguished thinkers as well. It is from them that the composer sourced some of the ideas he later realised in his work. 'We thought a lot of things over together, discussed much. It has been of great importance to me as a composer', he remarks [5, 169].

One of the many factors that can lead to the creation of a musical piece is quite simply an order. This is the way Beethoven's Wellington's Victory Or the Battle of Vittoria emerged, as well as the Tchaikovsky's overture 1812, Prokofiev's 5th Symphony and many other famous pieces. At the generating phase in this case, the composer's contacts widen perceptibly. Considering the sides that the composer has to come in contact with one might distinguish the individual and collective clients.

The former comprises the various patrons and sponsors, individual artists, impresarios, and the latter - the public, government and corporate and commercial structures such as publishing houses, opera and ballet or musical comedy theatres, etc. Sometimes various musical and cultural unions and societies, government and charitable organisations act as the clients ordering music from the composers. Of course, many other different organisations may act in the same way. The philharmonic societies with their great performing potential, the conservatories and musical colleges with their pedagogues who are often times very able performers, with their performing collectives, as well as the independent 
concert organisations, like symphony orchestras, choirs, ensembles, etc. may also act as the composers' clients.

The first group also includes a specific kind of patrons, the patrons whose role is not limited to the material or financial support of the composer by subsidising the creation of a certain piece (often times to remain in history as its sponsor). Sometimes the patrons become more intimate, more friendly with the creator and therefore become able to substantially influence the content of the piece which they help create. This is the way that the Russian Quartets by Beethoven emerged, supported by the Russian minister in Vienna A.K. Razumovsky. The generous and disinterested assistance of N.F. von Meck had not only enabled Tchaikovsky to compose some of his greatest work, like the 4th Symphony, the cycle of violin pieces, etc., but has also given rise to the vast, immensely important correspondence that explores the inner mechanisms of a great artist's creativity and reveals the essence of the communicative links that emerge during the creative process.

It is typical that the both cited groups also act as the popularisers of music or manage its functioning in a society. Therefore, when they order a piece from a composer they themselves, indirectly, realise the order of the wider public. And it is important, because the composers communicative field always implies a hypothetical, a model listener, since it is the listener, towards whom the whole process of communication is directed.

The leading role of the composer is characteristic of this phase of the communicative process development. It is he who initiates the contact, and the information exchange between the persons and organisations concerned is also carried out with his active participation and assistance. The leadership is preserved until the piece is transferred into the performer's sphere to be widely publicised. And if at the first stage the communicative flows were unidirectional, towards the composer, at the second stage it is a two-way communication - from the composer to the 'customers' (or performers) and back.

An opinion exists that initiating contacts with the 'customers' and performers is characteristic of the less known composers of limited talent, and established masters can expect both clients and performers to come to them. A study shows that it is much too often far from reality. A biographical research of the 35 composers with the highest listeners' rating has demonstrated that in spite of their popularity the composers allotted a considerable amount of their time for the contacts with the clients and the performers. It is noteworthy that most of the mentioned composers admitted their dependence on both of the latter, since they spent a great part of their creative careers searching for the acceptable objects and ways of communication.

At the same time, in spite of the considerable effort that J.-S. Bach put into the search for clients and publishers, only seven of his works were out of print in the course of his lifetime. This is very illustrative to the present discussion of the communicative links and the content of communication.
Thus, 'the silent conspiracy around his music, the povertystricken family, the abandoned grave - that is the gruesome triad of 'charities' that ... the greatest German of the 18th century had received' $[6,416]$. One might only note here, nevertheless, that even without the orders Bach wrote a great number of remarkable pieces...

What is said already should not leave an impression that a composer may be indifferent to the future of his creation. There are a vast number of examples that testify to the great care with which the composers approach the social being of their work. There is a very paradoxical excerpt by $\mathrm{H}$. Berlioz, 'Two years ago, while sleeping, I dreamed up a whole symphony. In the morning I was able to recall its first part, it was an allegro in A minor. I was at my desk already to write that down when I thought that if I put down the fragment I'd get carried away and go on writing $<\ldots>$ I would spend three to four months on this work alone. $<\ldots>$. Then, when the symphony is ready, I would give in to my copyist; I'd let it be copied and would thus make debts of 1000 to 1200 francs. When all the parts are copied I would be irresistibly tempted to hear my work performed. I would give a concert whose earnings would only cover half of my expenses. It would now be unavoidable. I would lose the little that I still possess. I shivered at the thought and put my pen aside <...>.' [7, 643-644].

In practice we encounter one more problem, the problem that might be termed as the selection of the aesthetic ideas by the society. The fact is that the communicative links and interrelations circulate much fewer musical works than is, in general, created. To precisely calculate the ratio between the works that are written and the works that are actually performed is impossible - there is no such statistics. But if we proceed from the point that the society only circulates 'original' musical ideas, the pieces that might be termed as actual aesthetic discoveries (L.A. Mazel), then the calculation might be conducted on the analogy with printed journalism. Thus A. Moles states that $98 \%$ of the annual press contains repeated information, whereas original information only appears in $2 \%[8,115]$.

Therefore one might suppose that it is approximately the same percentage of the musical creations that actively circulates through the communicative system. The remaining part quite simply fails to reach the public ear, partly because of its insufficient aesthetic merit. Plus, the great labour effort that has to be put into reproducing music, as well as the complicated character of the printing of musical texts have prompted the appearance of a very strict system of selecting the musical works.

In practice, to separate 'the wolves from the sheep' a micro-process of communication is used. At the stage when the piece is just created, it is first perceived in a group of professionals, it is not immediately that the wide public gets the chance of listening to it. The highly qualified musicians, musical editors, managers, theatre directors and other specialists form a kind of an expert group whose object is to evaluate the aesthetic potential of a piece and its possibilities for success. It is this group that makes a very important decision about whether it is worthwhile to let a 
certain piece be linked to the various channels through which music is publicised, that is, to the means of sociomusical communication.

Of course, this mechanism is far from perfect. And history knows a whole lot of instances when the experts were fatally mistaken, but there is no other, better way developed in the course of musical practice. The composers realise this, and, irrespective of their rating and popularity, usually try to present their work to the experts in a most favourable manner. Therefore, if any difference exists between the established and the lesser composers, it is contained in the level of prestige of the performers and customers that they interact with and in the time and effort that they have to put into reaching their goals. That is why, although the difference in opportunities is of considerable importance, no composer has been able to avoid the cares of the kind in course of his lifetime.

One has to note, of course, that the first performance is not only important pragmatically. To hear one's work performed, to feel the reaction of the prominent colleagues means to examine oneself and the correctness of one's own ideas expressed through sound, and, eventually, to 'fathom', to discern the possible future of one's creation.

And if during the initial communicative micro-process the piece has received favourable evaluation of the experts, it advances further to be published and propagated, and then, the third stage of the communicative process begins - the wide listening public gets familiarised with the musical piece.

The phase of presenting/exposition of the piece. For the piece to become accessible to a considerable number of listeners it has to be, as a rule, included into the repertoire of a considerable number of performers. That is why the authors are usually quite active in working to this end. At this point the composer encounters problems whose solution largely depends on the intensity of the communication between the participants of the whole process, on the quality of the links that sustain the communication, the quality that generally defines the level of the participants' interaction.

First of all, the composer often has to overcome the performer's doubts on whether the piece is going to be favourably accepted by the listeners and be popular with them. Then, the performer often doubts his own capability to express the author's idea in a suitable manner, since every new piece presents, so to speak, a complex equation with many variables. Therefore, for the composer, a certain new aspect of his communication with the performer arises. It is contained in his volition to variously assist the performer in getting comfortable with the new and unknown piece. For the performer, the study of a new piece is not only a joy of encountering and touching a new aesthetic value, but a risk of a certain kind. It is explained by the fact that the study of a new piece demands a great amount of time and artistic effort and often leads to a reduced care for the preservation and quality of the actual repertoire that is already being performed, the repertoire that has been variously tested in the performing practice already. And the performer has to approach this problem against the backdrop of his uncertainty as to where the work on the new piece would lead him.

Thirdly, the very thought of the considerable investment of time and energy that is required for the study of a new piece might turn, for the performer, into a certain psychological barrier in his contact with the composer. There's no doubt that when the performer sufficiently absorbs the new piece, when he surmounts the unavoidable technical difficulties and succeeds in finding a happy aesthetic resolution - his efforts are generously rewarded. Therefore, at this stage, the composer does his best to bring the performer around, or otherwise, he has to perform the music himself which invariably distracts him from his primary occupation - from composition - and consequently, narrows his communicative horizon.

One of the usual ways of attracting the performers' attention is the presentation of a piece in concert. The initiative here normally comes from the composer. Moreover, at first, he often has to organise the concerts himself, in different cities, on different stages, etc. Such an activity is often burdensome for the composer because of the financial, the psychological and organisational difficulties that arise in its course. And not every author is strong enough to overcome those difficulties. Sometimes the composers' patrons, who understand the situation very well, take the trouble of organisation upon themselves. Thus, for instance, when Tchaikovsky learned that in March 1879 E. Cologne was going to conduct the performance of his Tempest symphonic fantasia in Paris, he hurried to France. During the rehearsals and the concert itself he was perceptibly nervous for he could not fathom who the person was who had arranged the whole thing. N. Berberova wrote, 'Everything was meticulously rehearsed. The audience applauded and whistled with moderation. He saw Nadezhda Filaretovna in the box. She had her own plans for Colonne, she was thinking how much she should offer him for performing the Fourth symphony (by Tchaikovsky. A.Y.).' $[(9,136]$.

But the rare luck of having such a patron does not smile on every composer. Usually the authors have to fight hard for the life of their creation. Otherwise they might expect to be unknown, unnoticed and bitterly disappointed in themselves.

And the latter situation is unfortunately not that unusual. An example is described by Pablo Casals. The great 'cellist of the century has noted the absence of communicability on the part of the talented French composer Emanuel Moor the inability to contact with performers, to contain oneself, even the rudeness together with the aptitude to insult people - all that had finally shut the concert hall doors for him altogether. Even the repeated attempts by such prominent musician as Casals to help him could not set the situation right. Reminiscing on the case, the cellist had to admit that 'today Moor's work has practically succumbed to oblivion. $<\ldots>$ But this fact does not diminish its inherent value' $[10,101]$.

Sometimes various organisations come to the composers' rescue in solving the related problems. Thus the 
well known annual 'Moscow Autumn' festival organised by the Russian Composers' Union aims, among its other goals, to assist a greater number of performers in getting to hear the new work of the contemporary authors. It usually does not imply première performances, but has to do with presenting the pieces that had already been through the micro-process of communication but, for various reasons, had not acquired a sufficient number of performers. Needless to say, the range of the festival's objectives is much wider, but it also serves as a kind of a fair where the performers may choose the pieces that suit their aesthetic requirements.

We would also note that in the Western countries the musical festivals are often sponsored by the industrial concerns. Without trying to diminish the significance of such events, we have to say that the industrial companies look after their own interests - they need their public prestige to be raised and sustained.

But both the Western and the national festivals, if their practice is considered from the viewpoint of developing the theory of musical communication, have a number of drawbacks related to the problem of music selection, the principles and methods of that selection. The composer V. Dashkevich once said after a festival, 'I have no idea of the way one should approach the selection of the pieces for the festival. Any selection committee takes on an immense responsibility picking up the pieces of their own colleagues, sorting them into suitable, marginally acceptable and inadmissible. I do believe that no composer or musicologist has the right to judge so categorically. This is both unethical and hardly corresponds to the future of the new opera, the future that no one can foresee since the evaluation criteria are always relative' $[11,7]$.

Are their more democratic ways for a piece to find its performers? From the viewpoint of the theory of musical communication the advanced way would most likely be the one that would, on the one hand, make the system of publicising the notes of the musical pieces more simple and effective and, on the other hand, would possibly shorten the distance between the composer and the performer.

The appropriate attempts are now undertaken by businessmen in Russia and internationally. They have to do with the computer processing of the musical notation, the electronic means of music recording and the new technologies of music transmission and publicising. In fact the situation is connected with the communication firms that combine various functions: the notation type-setting, printing, acoustic recording, distribution, advertising and commercial activities.

In the Western countries such organisations that combine both commercial and cultural functions are called publishers. In the USA alone there exist 50,000 such companies (Data from the cited article by V. Dashkevich [see: 76]. These are small but active groups that invite authoritative performers to participate in the recordings or use audio or video tapes of the authors' performance. Their object is to acquaint the greatest possible amount of professional musicians and music lovers with the new pieces that the mentioned companies transmit and publicise through mass media. The companies send out notes, annotations to the new works, etc, on request. Each of such small publishing businesses 'receives a composer's licence for his works, each of them is interested in the pieces to be introduced in the best possible manner, to receive favourable publicity and press acclaim, to be performed again and again so that the publishers could cover their expenses and gain a profit' $[11,7]$.

An important part in attracting the performers' attention to a piece might be played by radio and TV broadcasts that include the composer's interviews together with the performance of his new pieces.

Quite often an author might use the important stimulus of dedicating a piece to its first performer as a token of gratitude. But it is not the way that always works. One might recall the well-known clashes related to Tchaikovsky dedicating his work. Thus the First piano concerto was originally dedicated to N. G. Rubinstein. But then the author and the performer quarrelled, the latter blamed the former for the fact that some passages in his work 'cannot be executed', so the composer dedicated his concerto to the German pianist Hans von Bülow who brilliantly performed it abroad. It was the same case with the violin concert: L. Auer considered it inexecutable, the other possible performer had been variously postponing the première $<\ldots>$ So Tchaikovsky had changed the dedication and the second issue of the score contained the name of the well known violinist Adolph Brodsky who was the first to perform it in Vienna.

Characterizing this phase of the communicative process one has to note that since the musical form is transferred to the performers, the composer starts to 'give' more to the society than he receives from it. The position of the composer in the communicative situation is also perceptibly changed: he gives, and the society represented by the performer receives. The communicative roles change. The society assumes the cumulative position and the composerthe generating one. But irrespective of any changes, the composer cannot feel completely satisfied since, sharing his ideas with the public and being largely ahead of the established public perceptions, he always risks encountering the failure to understand his work on the part of certain social groups. Throughout the whole history of the European musical culture one might single out just a very limited number of artists who could claim that they were satisfied by the public attitude to their creativity.

From this viewpoint it is important that the performers create a high standard of the performing musical form. At this stage the author has to oversee the realisation of his work in performance, much like an architect oversees the construction of the building that he designed. The author is present at rehearsals, suggests the correct variant of interpretation, and often takes part in selecting the key performers. After the piece has become an established part of the repertoire and certain performing traditions have been created, the author can, to a degree, let go of the initiative, allow it to commute to the performers. Let us now consider 
the ways the communicative processes develop in the other major sphere - the sphere of the reproduction of music in performance.

\section{THE PERFORMER'S SPHERE}

It has been mentioned above that music, as an art that exists and evolves in time and space, is impossible without the social act of its performance. And the performer is the very figure that 'enlivens' the music and brings it across to the listener in the course of the integral process of musical communication. The communicative processes that take place in the performer's sphere are largely similar to the processes that work in the sphere of the composer. But while the composer, as the creator of the form, directs his efforts to the search of the expressive means of the musical language that are most adequate to his original intention, the performer in his turn, has to search both for the expressive means that could best embody the author's concept, and for the means of getting his own aesthetic intentions across to the listener, as well as for the best ways of establishing adequate contact with the latter. Therefore the theory of musical communication may distinguish the three basic functions that the performer carries out: 1) the function of reproducing the musical piece; 2) the function of interpreting the aesthetic intention that the piece contains; and 3) the function of communicating that provides for the effective contact between the listener and the creator and his aesthetic ideas, as well as the creativity of the re-creator and the interpreter of the music - its performer.

The realisation of the three mentioned functions was historically formed in close connection with the evolution of music as an art, with the development of the public musical consciousness. Thus, the development of the musical art before the 15th century (that is, before Renaissance) was largely governed by the requirements of religion. Music often bore only applied significance, it had to be in accordance with the church ritual. Naturally, religion had elaborated its own conditions and norms for the musical communication, considerably influenced the development of genres, the style, etc. It is not by chance that the collective performance was rampant at that period (in particular, the a cappella choir singing). The musician had to assist the church in presenting the religious ideas to the parish. So, his functions were often reduced to the role of the 'animator' of the musical form. The situation with the temporal and folk music was quite different. In that case the social direction of the communicative processes was quite dissimilar from the religious music.

The performer starts to take on a completely different role in the Renaissance time. The development of a new culture in a society that proclaimed the independent value of the human individuality has prompted the art of solo performance and the appearance of the instrumental genres - the sonata and the concert. The public comes to be interested in the musician as the bearer of ideas. At the same time, the performer's activity is limited by a quite narrow circle of listeners, small concert halls (palace quarters, aristocratic salons) and not so frequent performances. And though the interpreter's function was not yet very actively manifested, the communicative role of the performer was already quite actively realised.

It is noteworthy that at that time the composers did not yet record their pieces on paper with detailed precision that implied further reproduction of every recorded sound. The performer was then successful not because he fully absorbed the original intentions of the author, but thanks to his talent for improvisation. At the same time, such a situation led to the lively and natural character of the very process of performing and therefore the communication itself acquired a living, dynamic development.

The emergence of the next stage of the communication between the performer and the listener is related to the establishment of the public concert as a form of performance, as well as to the formation of the national performing schools. The improvisation character of music performance loses its significance at this stage, since the composer now tries to fully record through notation the piece itself and the basic characteristics of its future performance in a way that he believes significant from the viewpoint of his original aesthetic intention. The profession of the performer becomes independent; the interpreting function becomes probably the most important one. The communicative links are thus deepened; they also acquire new content and significance.

All that leads to the situation when, for the experienced audiences, the performer's interpretation becomes quite as important as the piece itself. The very act of communication between the performer and the listener comes to be accompanied by a certain ritual, wherein the stage itself, the solemnity and preciseness of the whole arrangement, the limelight, a definite kind of the performer's costume, etc. take on certain significance. Therefore, some analysts believe that whereas earlier the music served a certain ritual, at this point the ritual comes to serve as the accompaniment to music [see $12,584-585 ; 100]$.

So, one might state that at that time the performer becomes the leader of the communicative process since he finds himself in the centre of communication that is realised along the triadic formula 'the composer — the performer the listener' and is supplemented by the two dual formulas - 'the performer-the composer' and 'the performer-the listener'.

As in the composer's case, the communicative process in relation to the performer evolves through three phases: 1) the phase of accumulating ideas (the performer assumes a cumulative position here); 2) the phase of the diverse 'communication' with the piece when the author's intention is deciphered, the text is memorised, the subtext is studied and the super-text created, and on that basis the expressive means are searched for, the means that are characteristic of this particular piece and its every performance (thus the performer assumes the position of generating ideas, of an aesthetic quest); and finally, 3 ) the phase of communicating with the audience (the position of exposing the author's and one's own ideas). And since those issues are quite minutely enlarged upon in the theory of musical performance [see, for instance, 13, 26-28, 67-72;151; 197, etc.] we deem it 
unnecessary to analyse them in great detail within the present work. Let us then get on to the listener's sphere.

Starting to discuss the issue, one has to first note the following: for a long time the communicative interaction between the performer and the listener was realised through a direct contact, at a concert, in a theatre, at a salon, etc. But the whole process was revolutionised by the invention of, first, the phonograph and then other means of sound recording, the actual, the living communicative link between the composer, performer and the listener was thus severed.

The appearance of the electronic means of music transmission has led to the distancing of the performer from the listener. Y.V. Kapustin believes that the introduction of the distance forms of music transmission has changed the very character of the performing art, as well as the character of the communication itself, since the direct contact with the listener was abolished and consequently the feedback, that is so important for the performer's productive activity disappeared. The student of the modern performing art justly notes that while the interpretation in the concert hall 'is, to a degree, the result of the mutual efforts of the performer and the audience, the result of their dialogue, at a radio, TV or a recording studio the performing process takes on the quality of a monologue' $[14,41]$. He believes that the appearance of independent, self-contained and at the same time quite a high-quality product of the performing art a 'record' of a performer's 'opus' of a kind that has taken on a material being limits the objectives of the act of performing.

At the same time, one has to note that the new possibility of distance communication through the materialised musical form opens a real opportunity for the further widening of the communicative field and the essential links within the spatial triad 'the composer-the performer-the listener'.

\section{THE LISTENER'S SPHERE}

The establishment of the process of communication in this sphere is also characterised by its triadic quality. Its first phase is connected with the cumulative position of the listener in relation to the composer and the performer. It has already been mentioned that perceiving the aesthetic ideas created by the composer and the performer, the listener does not only absorb them, but creates his own meanings for them in his mind. This is what, we believe, might be called the perceiving creativity of the listener.

It is well-known that the complex of individual characteristics as well as the quality of the products of his material and spiritual creativity depend, to a considerable degree, on the content and the quality of the information perceived. In this respect the content and the quality of the musical information that is circulated in the communicative channels take on a special significance - they act as a factor of the personality formation, as a means of influencing its spiritual activity.

Therefore by facilitating the consumption of quality information at the cumulative stage of the listener's sphere of the communicative process one might influence the development of an individual's musical consciousness and, eventually, the human creative activity in general. It is thus natural that the society tries to declare its orientation towards the consumption of the highest quality of information, which, in relation to music, implies the publicising of the academic genres - the so called serious music.

The information that the listener receives through the different channels is characterised by diverse content and orientation. We might accept the following basic scheme of its differentiation:

A. The general condition of the national and world musical culture, the most widely-utilised and valuable from the aesthetic and spiritual viewpoint styles, genres, forms of the serious music;

B. The topical part of the repertoire that was performed in a given musical area, the repertoire from which the listener selects something that most suits him, in respect of the authors, their particular pieces, the national and stylistic schools, etc.;

C. The knowledge of the basic stages of the development of music and some forecasts for its future progress (the feeling of the historical vertical);

D. The intensity of the musical advertisement and publicity;

E. The various information related to music gained on an everyday basis.

The basic channels of receiving such information that forms the spiritual and the evaluative approach to the musical art are contained, on the one hand, in the direct and indirect listening experience, on the other hand, in the musical education and upbringing - the factors that accompany the human (in their various kinds, forms and levels) through the entire course of his life.

These channels are exemplified in the various forms of familiarising oneself with music — from the mother's lullaby, musical education at kindergarten, the singing and music lessons at school, participating in different musical studios, ensembles, clubs, the studies at a musical school, performing music at home, etc. In general, the various complex channels of accumulating the information represent an activity that integrally influences the formation of a human being's musical consciousness.

Another form of accumulation of musical information is characterised by its at once active and passive quality. The form is contained in going to the concerts, attending theatre performances and various other musical events, watching musical films.

Then comes the passive form of accumulation. Consuming musical audio and video recordings is meant here. In this case the greatest influence upon the forming of the man's inner world is conducted through collecting records purposefully, exchanging them, etc.

The second phase of the communicative process in the listener's sphere is the reflexive phase. The information that is gathered here through the communicative channels, as it 
is being accumulated, forms a definite structure of evaluating the quality of the very information, facilitates the formation of such axiological categories as the aesthetic taste, the spiritual need, the musical interest. Using the integrity of this structure the listener may develop the skills of an active approach to the musical values, the approach that enables the listener to create his own vision of a musical piece upon hearing it. And we would like to stress that only such situation may give rise to the necessary conditions for the appearance of the feedback connection - for the opening of the channel of information that is so important for the integrity of the whole process for it makes its existence complete and sustained.

Dependence of the introspective activity of the mind upon the quality of the information perceived at the first stage of the communicative process is manifested in the level of adequacy phenomenon: only the spiritually charged, deep and complex information that the individual perceived may bring forth his high-level reflection and introspection in relation to the music perceived. One has to note, though, that we cannot identify the notion of the quality of introspection with the notion of the quality of human reactions and actions in general. Needless to say, that the human actions are not predominantly governed by the human reflections upon music. There are numerous other factors that influence the human motivation, and the reflection and introspection is just one of these. And the comprehension of the serious music by the listener also evolves along these lines.

At the third - the generating - stage, the listener establishes a kind of feedback with the performer and the composer, he enables the communication process initiated by the composer to be linked back, assists in deepening the process, acts as a certain criterion of its effectiveness. This communicative feedback is demonstrated in the appropriate reaction of the body of listeners in the concert hall, in the degree to which the certain musical performances and concerts are well-visited, in the letters from the music-lovers, their reaction reflected in the press, etc.

Besides, there is an indirect channel of the communicative feedback. It is contained in the fact that as the result of reflection upon the whole mass of the information received, the spiritual world of the human being is changed, transformed to a degree, a specific condition of purgation, or catharsis, as the Greeks termed it, arises. Consequently, all the subsequent acts of the human fall under the influence, including his attitude to art.

Besides the listener's communicative connection with the composer, the performer, the social musical culture in general, there is one more specific channel of communication that has to be mentioned here.

The channel of communication 'listener - listener' has long been in existence. It functions parallel to the basic channels 'the composer-the performer', 'the composer-the listener', 'the performer-the listener'. And the communication along this channel also may be conducted both directly and indirectly. The essential content of the musical communication within this channel lies in the mutual transfer, within the listening audience, of the individual reactions to the musical piece and its performance. A psychologically favourable atmosphere for the adequate perception of music is thus created, a feeling of something special about the occasion, a certain feeling of elevation and festivity arises this way, the whole event of perception takes on a certain significance, and that enables the interest to rise, the emotional sympathy to deepen, the psychic energy, so necessary for the deep and effective processing of the received musical information, to be accumulated.

The direct communication of this kind, that takes place in the time and space of the certain performance, means that the listeners are involved in the integral process - they exchange glances, they applaud, together they salute the artists at their appearance and thank them for their performance. These actions include the listeners into the general atmosphere of the concert that is charged by the many pulsing communicative links, and that charge defines the integral musical 'breath' of the concert hall. In this situation any non-musical move by a listener (a cough, a whisper, etc.) is perceived by the others as unnecessary static. Thanks to the collective perception and reaction, the functioning of all the communicative structures is optimised, in this case they become perceptibly different from those that work upon a solitary listener — and the whole thing eventually leads to a higher quality of music perception.

That is why the live perception of music in a concert or a theatre hall, the direct interaction of a community of listeners not only provides the most sound and vision information about the piece performed, the deep immersion into the event that is singular and unique, but that people live through together enables the perception to leave the more significant trace upon the listener's musical mind and spirit in general. And no other modern system of communication can begin to surpass this impact.

It is possible that the in the future the maximum 'effect of presence' would be imitated in the course of a radio or video broadcast. It is attempted today in cinema and television by including the close-ups of the listeners. But we believe that psychologically, the listener would never be able to be fully absorbed by the collective perception of music without the actual participation in the live process of musical performance in a concert hall.

The indirect communication (with a lag in time) implies that the interaction between the listeners may take place during a group or collective listening or watching the performance, as well as during the situation when the subsequent listener comes in contact with the listeners who were actually present at the concert - he may then perceive the atmosphere of the concert by hearing the applause, 'the breath of the audience', by different other indications, and when he watches the video he may also see the listeners' expressions and reactions and that helps in the restoration of 'the collective' element of the concert.

Another communicative channel is contained in the listeners' communication beyond the actual contact with the musical performance and its sound. One might mention here the discussion of the different impressions, the exchange of 
the audio and video records, of the information on the notes and books related to music, the opinions about the performers, composers, their records, etc. All that enables the sustained formation of the interests, preferences and tastes in music. These forms of communication related to music may, to a degree, influence the quality level of musical perception, the development of the musical consciousness of the whole society, that is, the result of the communication as a phenomenon of culture.

\section{The SPHERE Of ThE Musicologist-CritiC}

This sphere of communication is related to the evaluation, the research and regulation of the processes that evolve in the previous three spheres. The experience, the tendencies, the essential structures of the musical creativity accumulated by the individual creators and the performing collectives are analysed, generalised and partly projected into the future within the framework of this sphere. This enables a better preservation and transfer of the traditions, a more effective organisation of the whole musical process. Besides, the critic enters into the direct communicative contact with the composer, performer and the listener, and the musicologist interacts with the composer (or performer) or the listener in the course of discussions, lectures, public speaking.

Without the evaluation, the analysis and generalisation of the integral musical process, including the degree to which the communication therein is successful, it is impossible to comprehend the tendencies of the process development, to find the best ways to facilitate the process by activating its communicative links. The critic bears tactical functions here, functions that are more localised and pinpointed in time and space. The theoretical musicology globally encompasses all the aspects of the musical process in its historical perspective (that is, syntactically), as well as in its pragmatic aspect - in the integrity of all the components and links of the ensuing musical process.

Within the sphere of the musicologist-critic one may also distinguish a number of phases: the cumulative one, when the experience is accumulated, the traditions of studying and evaluating the musical process are learned, the historical and modern tendencies of the traditions' development are researched; then come the reflexive and the generating phases when the various phenomena of the musical life, as well as their perspective development, are considered and appropriate opinions are formed; then comes the presentation or exposition phase when the public expression takes place (through public speaking, the printed press and the electronic mass media), then the respective evaluation becomes known to the public and the most essential facts, events and tendencies of the musical process are singled out.

The criticism is closely connected with the problems of influencing the musical communication. The criticism might directly influence the musical creativity, performing and perception. But much too often this most important mission is not carried out in the best possible manner, the critics are often overly subjective, their opinions are influenced by stereotypes, superstitions, outdated traditions, their scope might be limited by their personal tastes, their ethnic, sociocultural and other preferences. History knows a considerable number of instances when the works of musical genius' were inadequately evaluated - it was the case with the cited instrumental concertos by Tchaikovsky, with Bizet's Carmen and Rossini's Il Barbiere du Seville, some works by Prokofiev and Shostakovich that only received their just dues a long time after their first performance.

That is why, in his projection of the future, the critic has to be, at least equal to the composer who is always ahead of his time and his listeners' perceptive capabilities. D.D. Shostakovich wrote on the issue, 'The modern music is complex, I agree. But were Beethoven and Moussorgsky, Scriabin and Prokofiev that simple, immediately understandable to their contemporaries? Many great creations are characterised by the complexity of thought and image, by an all-embracing philosophical idea that is expressed in a new and original form. One should not shun the complexity, but should rather be open to it, try to come out and comprehend it' $[15,10]$.

To make the first step towards everything new is the mission of critics and performers — those who professionally evaluate the aesthetic perfection of music, the degree to which it succeeded in discovering new expression for the ideal world of the good and beautiful.

\section{The Metastructural LEVEl OF COMMUNICATION}

This level is external in relation to the basic structures of the musical process. It basically deals with non-specific information, providing for the connection of the specific field of the musical art with the wider area of socio-cultural phenomena - the spiritual structures of the society, such as the public consciousness, religion, the theoretical and practical forms of thought (including those related to mythology, philosophy, etc.).

Through the communication links with these areas the music absorbs a wide cultural, social, everyday life context; these links empower the most important feedback circuit that enables the information to circulate in the closed system of musical art. In its turn, the active direction of the communicative process from the composer and the performer to the listener leads the music into the open, into the society where it influences the consciousness of the individual and the society as a whole, assisting, to a degree, in its 'theorisation', in stimulating the human aesthetic potential, fantasy, the development of the ability to create ideal models of the man and the world and to act according to these models.

This level is considerably different from the microstructural and the structural levels, both in the character of the information that circulates within it and in the ways of the information transfer. And while the two latter levels are basically related to the specific mechanisms of re-encoding information, the mechanisms that enable the isomorphic reproduction of the composer's original meanings, the highest, the meta-structural level has to do with the bigger structural units of the musical process - the patterns, norms, 
traditions, the genres, styles and other typological entities that are oriented to a definite way of perception formed by the social practice, to the established concert and other forms of performing music, etc.

These are the communicative structures that are most suitable for the transfer through the open channels that connect the music to the reality of life, the wide cultural field that includes the other arts as well. This is the channel through which the transfer and absorption of the musical experience of the society is conducted in pedagogics, the musical and aesthetic education, in the publicising of art, etc.

Besides, the meta-structural level possesses a hidden communicative channel that provides for the contact of the composer's and performer's aesthetic thought with the higher levels of the listener's spiritual experience, connecting them to the sphere of the public consciousness, to the structures of the aesthetic processing of the actual reality, to the cultural levels accumulated through history, to the unlimited creative capabilities of man.

At the meta-structural level one cannot differentiate sharply structured phases of the communicative process. They are rather vague and overlapping. We might note the cumulative phase - the accumulation of the theoretical and practical experience, the development of the material basis in relation to music, including the fund of the musical values - the notes, films, audio and video recordings, the musical instruments, the different equipment for the concerts, for the printing of notes, etc; the traditions of performing and educating performers; the search for the active forms of communication with the listener; the accumulation of the traditions, forms and skills of perceiving music, its evaluation by the critics and its study by performers, etc.

The reflexive phase might include all the kinds and forms of the social evaluation of the musical process, its participants and phenomena, the results of the critics' and musicologists' activity, the defining of the listeners' preferences, of the composers' 'rating' with the performers, as well as other evaluative structures, including the theoretical support of the most effective means of creative and educational activity in music.

The generating phase would then include the traditions that are formed in a society, the norms and forms of the interaction with music, the degree of the prestige of music and musicians in the society, the founding of the concert halls, musical theatres, educational institutions, museums, musical collectives, the manufacturing of the musical instruments and other actions that find support in the public consciousness and practical life, as well as the permanently active structures, such as publicity, advertising, sponsorship, management and many others.

The meta-structural level is the one where the most suitable and, to a degree, the most effective ways are open for the society and its institutions to influence the different communicative flows that take place in the musical art. The influence that is related to the generating phase has, by all means, to take into consideration the complex, diverse and interrelated communicative structure of music, since the influence upon each of the links of the integral process may lead to inadequate results. History demonstrates that the incompetent, rude and sometimes malicious interference into the musical process always has a detrimental effect upon culture, hindering the progress of the musical art development.

At present, the communicative spheres of the composer and the performer are more or less well-studied, which cannot be said of the listener's sphere. Little attention was also given to date to the exploration of the communicative processes, their complex structure and functional significance in the sphere of musicology and criticism, and that was, up to recent times, primarily connected with ideological and political reasons, which T.A. Kurysheva justly notes in one of the few monographs dedicated to this important issue [see 16].

Today one might note that the attention to the studies in this particular field of the musical theory has grown, but it is yet focused upon the factual data from a given historical period. The theoretical research of this particular sphere of the processes of musical communication is probably a matter of the future.

Summing up some of the results gained in the course of the analysis of the structure of the process of musical communication we should stress again that the singling out of the central - structural - level that embraces all the four spheres - the composer's, the performer's, the listener's and the musicologist/critic's - is not at all artificial or unwarranted. The appropriate addition of the fourth participant to the established triad 'the composer-the performer-the listener' enables a more thorough analysis of the channels that connect these communicative spheres, the channels generating, encoding and decoding, transforming and evaluating the aesthetic information, it also provides an opportunity to clarify the role of each participant of the musical communication in the general complex process of the music's existence.

The distinguishing of the micro-structural levels in all the spheres of the musical communication provides for a clearer understanding of the stages of the interaction with the aesthetic meanings and codes, it also gives an opportunity to gain a more precise idea of the stages of the information transformation and of the role of the communicative flows at each of the given stages. It turns out to be quite natural that in the composer's and the performer's spheres the sequence of the three process stages is similar (although there are obvious differences in the original material itself), and that similarity leads to the analogous character of their creative thinking aimed at producing an aesthetic value.

In the listener's sphere the triad of the basic phases seems to be preserved, but the purpose content of the phases is slightly different here. Here the process develops from the accumulation of information to reflection and further to generating both musical and extra-musical active and creative meanings that then come out into the person's individual behaviour. This fact makes it possible to 
distinguish still another phase here, that partly transgresses into the meta-level - the phase of the impact of the musical information (or a post-phase).

It turned out that the musicologist-critic proved to be a figure that influences all the components and aspects of the communicative process. But here one might also single out some micro-structural phases that are largely akin to those of the listener, but that have to do with a slightly different material and direction, that also progress with a higher creative intensity.

Thus, within the central structural level a certain binary opposition of the spheres arises: on the one hand, there are the creators of the aesthetic values; on the other — those who perceive and evaluate the work of the former. The latter are impacted by the contact with music and bring their impressions into the other spheres of activity; the listener into everyday life, the musicologist-critic — into the creation of theoretical and critical works.

The diverse information flows that circulate at the structural and micro-structural level come forth into the meta-structural level of the public musical consciousness and creative activity in its widest sense. That is the level at which the most important feedback circuit of the whole communicative process is closed, and that enables the musical information to enter the spiritual life of a society and influence the human activity, the development of the highest forms of the human thinking.

It is here, too, that the vast amount of cultural and other information is linked to the channels of the musical communicative structure, charging it with energy, providing it with new meanings, forms, sharing all the treasures of human thought and feeling. We believe that the metastructural level opens a possibility for a certain influence upon the integral development of the whole process of musical communication and its separate components, as well as upon the multifarious processes of the musical art in general.

The basic structural regularities of the musical communication considered here are presented (not quite fully and, of course, very relatively) in a graphic scheme [3, 270]. The scheme may, to a degree, give a more clear idea of the complex and diverse structure of the system of musical communication.

\section{CONCLUSION}

Summing up all the above, we may say that all the three spheres - the composer's, the performer's and the listener's are connected between each other and cannot exist without each other. Thus, the composer's creations are meant for the listener and the performer and the last ones need compositions to interact.

\section{REFERENCES}

[1] Tsypin G.M. Music Activity Psychology, - Moscow, 1994.
[2] Delson V. The Problems of Skryabin's and Prokofiev's Piano Compositions Performance.// The Issues of Music Performing Art.Moscow, 1962.

[3] Rachmaninov S.V. Letters. Moscow, 1955.

[4] Tchaikovsky P.I. Literature works, correspondence.// Complete works.- Moscow, 1962-63.

[5] Tsypin G.M. A person, a talent, a labour. - Moscow, 1994.

[6] Rosenshield K. The History of Foreign Music. - Moscow, 1976.

[7] Berberova N. Tchaikovsky: The History of One Life. - SpB, 1993.

[8] Moles Abraham. Cultural and Social Dynamics. Tr. from French. Moscow, 1973

[9] Belyaeva-Exemplyarskaya S. A Study on Modern Mass Listener Psychology.// Music Education, 1929. \# 3-4.

[10] Kan A. Joys and Sorrows. Pablo Casalis`s Considerations, Confessed to Albert Kan. - Moscow, 1977.

[11] Dashkevich Vl. Coming to the Author's Rights Society. //Music Academy. 1992. \#4

[12] Yampolsky Igor. Music Performance// Music Encyclopaedia. Moscow, 1974.

[13] Alexev A.D. Interpretation as an Art. On Methodological Analysis of the Problem.// Music Education in the Context of Culture. \#1., 'Moscow, 1994.

[14] Kapoustin Yu.V. A Musician Performer and His Audience.Leningrad, 1985.

[15] Shostakowich D. Music and Time: Composer's Notes.// Music and Modern Time. -Moscow, 1976.

[16] Kurisheva T. A Word About Music. On Music Critics and Music Journalism: Essays. - Moscow, 1992. 